\title{
Can corneal pannus with trachomatous inflammation - follicular be used in combination as an improved specific clinical sign for current ocular Chlamydia trachomatis infection?
}

Tamsyn Derrick ${ }^{1 *}$ (D), Martin J. Holland ${ }^{1}$, Eunice Cassama ${ }^{2}$, Rod Markham-David ${ }^{1}$, Meno Nabicassa ${ }^{2}$, Michael Marks ${ }^{1}$, Robin L. Bailey ${ }^{1}$ and Anna R. Last ${ }^{1}$

\begin{abstract}
Background: Trachoma is a blinding disease caused by conjunctival infection with Chlamydia trachomatis (Ct). Mass drug administration (MDA) for trachoma control is administered based on the population prevalence of the clinical sign of trachomatis inflammation - follicular (TF). However, the prevalence of TF is often much higher than the prevalence of $\mathrm{Ct}$ infection. The addition of a clinical sign specific for current ocular Ct infection to TF could save resources by preventing unnecessary additional rounds of MDA.

Methods: Study participants were aged between 1-9 years and resided on 7 islands of the Bijagos Archipelago, Guinea Bissau. Clinical grades for trachoma and corneal pannus and ocular swab samples were taken from 80 children with TF and from 81 matched controls without clinical evidence of trachoma. Ct infection testing was performed using droplet digital PCR.

Results: New pannus was significantly associated with $C$ infection after adjustment for TF $(P=0.009, \mathrm{OR}=3.65$ (1.4-9.8)). Amongst individuals with TF, individuals with new pannus had significantly more Ct infection than individuals with none or old pannus ( $75.0 \%$ vs $\left.45.5 \%, C_{i}^{2} P=0.01\right)$. TF and new pannus together provide a highly specific $(91.7 \%)$, but a poorly sensitive $(51.9 \%)$ clinical diagnostic test for $\mathrm{Ct}$ infection.

Conclusions: As we move towards trachoma elimination it may be desirable to use a combined clinical sign (new pannus in addition to TF) that is highly specific for current ocular Ct infection. This would allow national health systems to obtain a more accurate estimate of Ct population prevalence to inform further need for MDA without the expense of $\mathrm{Ct}$ molecular diagnostics, which are currently unaffordable in programmatic contexts.
\end{abstract}

Keywords: Trachoma, Pannus, Chlamydia trachomatis

\footnotetext{
* Correspondence: tamsyn.derrick@lshtm.ac.uk

${ }^{1}$ Clinical Research Department, Faculty of Infectious and Tropical Diseases,

London School of Hygiene and Tropical Medicine, London, UK

Full list of author information is available at the end of the article
} 


\section{Background}

Trachoma is the most common infectious cause of blindness worldwide and is initiated by conjunctival infection with Chlamydia trachomatis (Ct). Children living in trachoma-endemic communities suffer repeated infections with $\mathrm{Ct}$, which manifests as a follicular conjunctivitis (trachomatous inflammation - follicular (TF)), often accompanied by papillary inflammation (trachomatous inflammation - intense (TI)). Repeated Ct infections can result in fibrosis and scarring of the tarsal plate, leading to in-turning of the eyelashes (trichasis), abrasion of the cornea and eventually blindness. It is currently estimated that $\sim 21.4$ million people have active trachoma [1], many of whom will go on to develop the scarring sequelae that can lead to blindness.

The World Health Organization (WHO) aims to achieve global elimination of trachoma as a public health problem by the year 2020 through implementation of the "SAFE strategy" (Surgery for trichiasis, Antibiotics for Ct infection, Facial cleanliness and Environmental improvements to prevent infection) [2]. When the baseline prevalence of TF in 1-9 year olds $\left(\mathrm{TF}_{1-9}\right)$ is between $10-29 \%$ the WHO recommends that 3 years of annual mass drug administration (MDA) with Azithromycin should be delivered, extending to 5 years when $\mathrm{TF}_{1-9}$ is $\geq 30 \%$. After 3 or 5 years of MDA, impact surveys should be conducted before deciding whether to continue or stop treatment [3]. MDA should be conducted alongside the complementary S, F and $\mathrm{E}$ activities. To meet elimination targets, $\mathrm{TF}_{1-9}$ is recommended to be below $5 \%$ at the district level. If a district has $\mathrm{TF}_{1-9}$ between 5-10\%, within-district communities should be assessed and treated separately by the same principles and $\mathrm{F}$ and $\mathrm{E}$ should continue.

The use of TF as a clinical sign for Ct infection can however be misleading. In low prevalence areas there is discordance between the prevalence of TF and $\mathrm{Ct}$ infection [4-7], particularly after the introduction of MDA [8-10]. Clearance of TF lags behind the clearance of Ct infection on both individual and population levels $[8,11,12]$. It is thought that other chlamydial species and non-chlamydial bacteria can contribute to the presence of TF in lowprevalence settings, although the impact of these infections on scarring development is unclear [13-17]. These observations have led to the suggestion that unnecessary additional rounds of MDA may be delivered by resource poor national eye health programs. This was illustrated by a recent study in The Gambia, where after one round of MDA no ocular infections with $\mathrm{Ct}$ were detected in the 1-9 yr olds in the communities under study. This enabled a premature halt to the three year treatment regime in all communities in that treatment arm [18]. Follow up at 3 years showed that TF and $\mathrm{Ct}$ infection prevalence were equivalent between communities where MDA ceased after a single round of treatment and in those that had received MDA for 3 years [18]. There are significant costs associated with molecular testing for $\mathrm{Ct}$ however, such that cost savings may only be achieved if testing just a sample of children in each census area or in a sample of census areas [19]. Implementation of a simple low-cost $\mathrm{Ct}$ diagnostic assay that can be used in regional health care facilities to obtain $\mathrm{Ct}$ infection population prevalence data is desirable, however testing is currently confined to expensive commercial platforms, which without subsidies or donations are not feasible in operational settings.

Alternatively, extra information relating to the presence of ocular $\mathrm{Ct}$ infection in the community could be ascertained by the inclusion of additional clinical signs that are more sensitive or specific for current ocular infection with $\mathrm{Ct}$, such as corneal pannus. Pannus is the sub-epithelial neovascularization of the peripheral cornea. Corneal pannus was once one of three cardinal clinical signs considered to be essential for the diagnosis of trachoma (alongside "granulations" (follicles) and "conjunctival scarring") [20-22], however it has now been discarded in the routine diagnosis of trachoma and was not included in the WHO simplified grading system [23]. It was noted that corneal pannus was rarely seen independently and was more often seen alongside follicles or scarring [20], suggesting that pannus is rarely non-specific and that its accompaniment to the two other signs of trachoma could indicate the presence of $\mathrm{Ct}$ infection. In this small-scale study we have revisited the utility of pannus as a diagnostic tool that might increase the concordance of clinical signs and current ocular Ct infection.

\section{Methods}

\section{Ethical, consent and permissions}

The study was conducted in accordance with the declaration of Helsinki and was approved by the Ethics committee of the London School of Hygiene and Tropical Medicine, The Gambian Government/Medical Research Council Unit and the Comité Nacional de Ética em Saúde of Guinea Bissau. Written informed consent was obtained from a parent or guardian prior to a participant's enrollment in the study.

\section{Clinical sample collection and study design: case control} Samples were collected from children aged from 1 to 9 years residing on 7 islands of the Bijagos Archipelago, Guinea Bissau, 6 islands of which were trachoma treatment naïve at the time of sample collection. Children were screened for clinical signs of trachoma by a trained clinician using the WHO simplified trachoma grading system [23] and the WHO 1981 FPC trachoma grading system [24]. Individuals presenting with follicular scores 
of "F2" or "F3" using the FPC system were equivalent to "Trachomatous Inflammation - Follicular (TF)" using the simplified system and were defined as "TF cases". Individuals with no clinical signs of follicles (F0), papillary hypertrophy $(\mathrm{P0})$ or conjunctival scarring $(\mathrm{C} 0)$ were enrolled as "controls" and were matched to TF cases by age, gender and village. Pannus was graded in all TF cases and controls enrolled in the study as not present, new (active neovascularization) or old (empty or scarred vessels). Macro photographs were taken of the edge of the cornea using a Nikon D60 SLR camera with a VR AF-S micro Nikkon $105 \mathrm{~mm}$ 1:2.8G ED lens. In this study we evaluated the use of corneal pannus as a clinical sign for Ct infection using field observed grades, analogous to how it would be implemented by national trachoma control programmes.

Two conjunctival samples were taken from the left upper tarsal conjunctiva of each participant with Dacron swabs (Puritan) using standard methodology [10, 25]. Swabs were stored in RNAlater (Life technologies) and kept in a cold chain in the field before subsequent storage at $-80{ }^{\circ} \mathrm{C}$. The two swabs from each individual were pooled for RNA and DNA extraction, which was carried out using Norgen total RNA and DNA purification kits following the manufacturers instructions (Norgen Biotek).

\section{Molecular testing for Chlamydia trachomatis}

Droplet-digital PCR (ddPCR) was used to diagnose $\mathrm{Ct}$ infection, as described previously $[26,27]$ with the following modifications. Forward and reverse primers were used at $0.9 \mu \mathrm{M}$ and probes were used $0.2 \mu \mathrm{M}$. Eight microlitres of sample DNA was added to each $20 \mu \mathrm{l}$ reaction. An initial diagnostic assay detecting $\mathrm{Ct}$ plasmid and human RPP30 endogenous control was carried out on all samples. Ct chromosomal gene $o m c B$ and plasmid DNA were then quantified in positive samples to determine $\mathrm{Ct}$ load. Primer and probe sequences are described elsewhere [27]. Thermal cycling conditions were as follows: $95{ }^{\circ} \mathrm{C}$ hold for $10 \mathrm{~min}$, followed by 40 cycles of $95{ }^{\circ} \mathrm{C}$ for $10 \mathrm{~s}$ and $60{ }^{\circ} \mathrm{C}$ for $20 \mathrm{~s}$ with a final hold of $98{ }^{\circ} \mathrm{C}$ for $12 \mathrm{~min}$. ddPCR was performed on a Bio-Rad QX100 Instrument and data were collected using Quantalife software (Bio-Rad). Copy number was calculated in R using previously published scripts [26].

\section{Statistical analysis}

Data were analysed in R (www.R-project.org). Multivariable logistic regression was performed with $\mathrm{Ct}$ infection as a binary outcome and clinical signs of TF and pannus were included as independent variables. Age and gender were included a priori.

\section{Results}

Clinical phenotypes of study participants are shown in Table 1. Eighty individuals had TF, of whom 36 (45\%) had new pannus. Amongst individuals with TF, individuals with new pannus had significantly more Ct infection than individuals with none or old pannus $(75.0 \%$ vs $45.5 \%$, Chi $\left.^{2} \mathrm{P}=0.01\right)$. Nine of the 81 controls $(11.1 \%)$ and 2 of the $5 \mathrm{Ct}$ positive controls had new pannus. Representative photographs of pannus grades are shown in Fig. 1. Chlamydial load increased with TF grade (data not shown), but there was no apparent relationship between pannus and Ct load (Table 1).

In multivariable logistic regression analysis $\mathrm{TF}$ was strongly associated with $\mathrm{Ct}$ infection $\left(P=1.16 \times 10^{-7}\right.$, $\mathrm{OR}=18.17$ (6.73-59.5)). New pannus was independently associated with $\mathrm{Ct}$ infection after adjustment for TF $(P=0.009$, OR $=3.65(1.4-9.8))$. Old pannus was not associated with $\mathrm{Ct}$ infection $(P=0.859, \mathrm{OR}=0.89$ (0.23-3.26)) (Table 2). Exclusion of the 31 individuals from the MDA treated island did not significantly change the results of the regression analysis; TF $\left(P=3.83 \times 10^{-6}\right.$, $\mathrm{OR}=13.56(4.83-45.7))$ and new pannus $(P=0.01$, OR $=$ 4.04 (1.43-11.96)) remained significantly associated with current $\mathrm{Ct}$ infection (data not shown).

We investigated the sensitivity and specificity of new pannus and TF as a diagnostic clinical sign for Ct infection. TF was more sensitive than new pannus $(90.4 \%$ for TF v $55.8 \%$ for new pannus), whereas new pannus was more specific than TF (69.7 \% for TF v $85.3 \%$ for new pannus). The sensitivity of using new pannus and TF together to diagnose $\mathrm{Ct}$ infection (excluding individuals presenting with new pannus without TF, or TF without new pannus) was $51.9 \%$ and the specificity was $91.7 \%$

Table 1 Clinical phenotypes of study participants

\begin{tabular}{|c|c|c|}
\hline & Controls & TF cases \\
\hline Total & 81 & 80 \\
\hline$\%$ Female & $58 \%$ & $58.75 \%$ \\
\hline Median age (IQR $\left.{ }^{\mathrm{b}}\right)$ & $4(3-6)$ & $4(2-6)$ \\
\hline Ct positive (\%) & 5 (6.2\%) & 47 (58.8 \%) \\
\hline Ct load ${ }^{\mathrm{a}}\left(\mathrm{IQR}^{\mathrm{b}}\right)$ & $345(42-1077)$ & $4600(656-16462)$ \\
\hline No pannus & 55 & 34 \\
\hline Ct positive (\%) & $2(3.6 \%)$ & 15 (44.1\%) \\
\hline Ct load ${ }^{\mathrm{a}}\left(\mathrm{IQR}^{\mathrm{b}}\right)$ & 36672 (18509-54836) & 4500 (1006-6112) \\
\hline Old pannus & 17 & 10 \\
\hline Ct positive (\%) & 1 (5.9\%) & $5(50 \%)$ \\
\hline Ct load $\left(I Q R^{b}\right)$ & 1078 & $41250(432-46750)$ \\
\hline New pannus & 9 & 36 \\
\hline Ct positive (\%) & $2(22 \%)$ & $27(75 \%)$ \\
\hline Ct load ${ }^{\mathrm{a}}\left(\mathrm{QQR} \mathrm{R}^{\mathrm{b}}\right)$ & $31(25-36)$ & $6800(642-16462)$ \\
\hline
\end{tabular}

${ }^{\mathrm{a}} \mathrm{Ct}$ load = median omcB copies/swab in samples positive for Ct DNA by ddPCR ${ }^{\mathrm{b}} \mathrm{QQR}=$ Inter-quartile range 


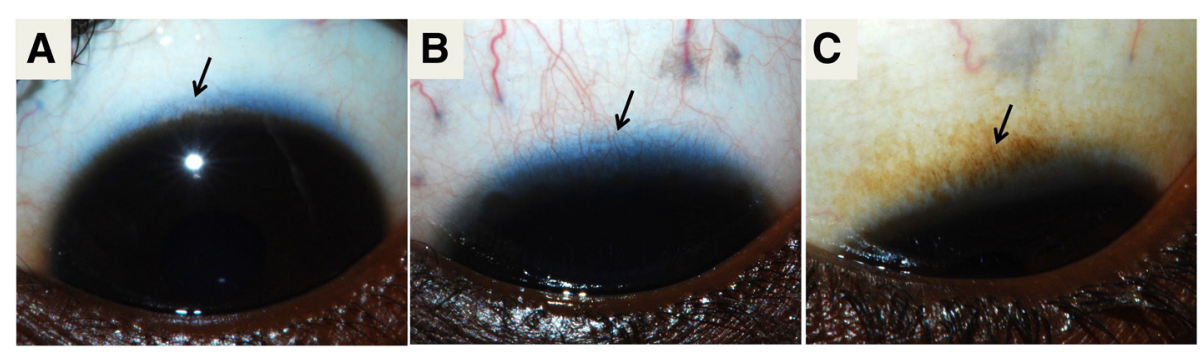

Fig. 1 Clinical grading of corneal pannus: example photographs. a Normal healthy cornea, b new pannus and c old pannus. Arrows point to the lack of vascularization in controls $\mathbf{a}$, neovascularization in new pannus $\mathbf{b}$ and old scarred vessels that indicate old pannus $\mathbf{c}$

(Table 3). TF and new pannus together had the highest positive predictive value (PPV) $(75 \%)$ and a good negative predictive value (NPV) $(80 \%)$.

\section{Discussion}

We have reported the performance of new pannus in combination with TF as a clinical diagnostic test for ocular $\mathrm{Ct}$ infection. New pannus was independently associated with $\mathrm{Ct}$ infection after adjustment for TF, suggesting that use of the two clinical signs together might increase the likelihood of detecting $\mathrm{Ct}$ positive individuals. The presence of TF and new pannus together was a more specific diagnostic test for $\mathrm{Ct}$ infection than either alone but it was much less sensitive; therefore use of this combined clinical sign in a trachoma control programme is likely to underestimate the prevalence of $\mathrm{Ct}$ infection.

The performance of TF alone as a clinical diagnostic test for $\mathrm{Ct}$ infection in these samples was perhaps overinflated by the study design. A population-based survey carried out on four other treatment-naïve islands of the Bijagos Archipelago in Guinea Bissau in 2012 found that in 1-9 year olds, the prevalence of TF was $22 \%$ and the prevalence of $\mathrm{Ct}$ infection was $25 \%$ [25]. This present study had a case-control design and participants were enrolled on the basis of clearly defined clinical phenotypes, therefore the likelihood of individuals with TF having $\mathrm{Ct}$ infection was high. These are limitations of this study and in order to assess the use of new pannus and $\mathrm{TF}$ in combination as a proxy for current $\mathrm{Ct}$

Table 2 Multivariable logistic regression of the relationship between clinical phenotypes and Ct infection

\begin{tabular}{lll}
\hline & $P$ value & ${ }_{a} \mathrm{OR}^{\mathrm{a}}\left(2.5-97.5 \% \mathrm{Cl}^{\mathrm{b}}\right)$ \\
\hline TF & $1.16 \times 10^{-7}$ & $18.17(6.73-59.5)$ \\
Pannus: New & 0.0088 & $3.65(1.4-9.8)$ \\
Pannus: Old & 0.859 & $0.89(0.23-3.26)$ \\
Gender (Male) & 0.738 & $0.86(0.35-2.08)$ \\
Age (years) & 0.053 & $1.23(1-1.52)$
\end{tabular}

Model AIC (Akaike Information Criterion) is 143.46

${ }_{a}$ OR: Adjusted Odds Ratio (adjusted for all other variables in the model as listed)

${ }^{\mathrm{b}} \mathrm{Cl}$ Confidence Interval infection in an elimination context this experiment should be repeated in a larger population-based survey in a low-prevalence area $\left(\mathrm{TF}_{1-9}\right.$ between $\left.5-10 \%\right)$. It is possible that in a low-prevalence area the sensitivity of TF and new pannus together as a diagnostic tool for $\mathrm{Ct}$ infection would be improved relative to TF alone.

\section{Conclusions}

In communities where the prevalence of $\mathrm{TF}$ and $\mathrm{Ct}$ infection are known or suspected to be discordant, it may be desirable to use a more specific clinical diagnostic sign for Ct infection in order to prevent an overestimation of the disease burden. The poor sensitivity of the test however renders it inappropriate in a programmatic context. There were significantly more $\mathrm{Ct}$ positive individuals with both TF and new pannus compared to individuals with $\mathrm{TF}$ alone, therefore a cost-efficient approach for assessing whether to prematurely stop MDA in a community might be to perform molecular Ct testing only on samples from individuals presenting with both clinical signs. Early implementation of the 'stopping rule' based on testing a sample of individuals most likely to have Ct infection, or, as we move towards elimination, treating just this sample of individuals most likely to have Ct infection, could lead to significant cost-savings without loss of treatment impact.

\section{Consent to publish}

Written informed consent was obtained to publish anonymized individual-level clinical data and images.

Table 3 Performance of TF and new pannus as a diagnostic test for Ct infection

\begin{tabular}{llcc}
\hline & TF & New pannus & TF \& new pannus \\
\hline Sensitivity & 90.4 & 55.8 & 51.9 \\
Specificity & 69.7 & 85.3 & 91.7 \\
PPV & 58.8 & 64.4 & 75.0 \\
NPV & 93.8 & 80.2 & 80.0 \\
\hline
\end{tabular}




\section{Abbreviations}

Ct: Chlamydia trachomatis; TF: Trachomatous inflammation - follicular; MDA: Mass drug administration; PPV: Positive predictive value; NPV: Negative predictive value; AIC: Akaike information criterion.

\section{Competing interests}

The authors declare that they have no competing interests.

\section{Authors' contributions}

TD collected data in the field, performed all laboratory work and analyses and drafted the manuscript. MH contributed to data analyses and revised the manuscript. EC collected samples and data in the field. RMD collected data and took clinical photographs in the field. MN assisted with coordination and logistics of the field study. MM assisted with data analyses and edited the manuscript. RB trained the clinical grader and RB, MH and AL conceived and designed the study. AL performed clinical grading and sample collection, assisted with data analyses, edited the manuscript and coordinated the field study. All authors read and approved the final manuscript.

\section{Acknowledgements}

The authors would like to express great thanks to the study participants and to the field team. This study was funded by grants from the Wellcome Trust (093368/Z/10/Z and 097330/Z/11/Z) and Fight for Sight in the form of a graduate studentship for TD (http://www.wellcome.ac.uk;http://www.fightforsight.org.uk). MM is supported by a Wellcome Trust Clinical Research Fellowship (102807/Z/13/Z).

\section{Author details}

${ }^{1}$ Clinical Research Department, Faculty of Infectious and Tropical Diseases, London School of Hygiene and Tropical Medicine, London, UK. ${ }^{2}$ Programa Nacional de Saude de Visao, Ministerio de Saude Publica, Bissau, Guinea Bissau.

Received: 13 November 2015 Accepted: 15 January 2016

Published online: 27 January 2016

\section{References}

1. Papyrus E, States U. Weekly epidemiological record Relevé épidémiologique hebdomadaire. 2012. p. 161-8.

2. Future approaches to Trachoma Control: Report of a Global Scientific Meeting, Geneva, 17-20 June 1996. World Heal Organ 1997. Unpublished document WHO/PBL/96.56, available at http://apps.who.int/iris/handle/ $10665 / 63413$ ?locale $=$ en

3. World Health Organisation. WHO Report on the 3rd Global Scientific Meeting on Trachoma. 2010.

4. Schachter J, West SK, Mabey D, Dawson CR, Bobo L, Bailey R, et al. Azithromycin in control of trachoma. Lancet (London, England). 1999; 354:630-5.

5. Burton MJ, Holland MJ, Faal N, Aryee EAN, Alexander NDE, Bah M, et al. Which members of a community need antibiotics to control trachoma? Conjunctival Chlamydia trachomatis infection load in Gambian villages. Invest Ophthalmol Vis Sci. 2003:44:4215-22.

6. Wright HR, Taylor HR. Clinical examination and laboratory tests for estimation of trachoma prevalence in a remote setting: what are they really telling us? Lancet Infect Dis. 2005;5:313-20.

7. Harding-Esch EM, Edwards T, Sillah A, Sarr I, Roberts CH, Snell P, et al. Active trachoma and ocular Chlamydia trachomatis infection in two Gambian regions: on course for elimination by 2020? PLoS Negl Trop Dis. 2009;3:e573.

8. Solomon AW, Harding-Esch E, Alexander NDE, Aguirre A, Holland MJ, Bailey $\mathrm{RL}$, et al. Two doses of azithromycin to eliminate trachoma in a Tanzanian community. N Engl J Med. 2008;358:1870-1.

9. Burton MJ, Holland MJ, Makalo P, Aryee EAN, Sillah A, Cohuet S, et al. Profound and sustained reduction in Chlamydia trachomatis in The Gambia: a five-year longitudinal study of trachoma endemic communities. PLoS Negl Trop Dis. 2010. doi:10.1371/journal.pntd.0000835

10. Keenan JD, Lakew T, Alemayehu W, Melese M, Porco TC, Yi E, et al. Clinica activity and polymerase chain reaction evidence of chlamydial infection after repeated mass antibiotic treatments for trachoma. Am J Trop Med Hyg. 2010;82:482-7.
11. BAILEY R, DUONG T, Carpenter R, WHITTLE H, Mabey D. The duration of human ocular Chlamydia trachomatis infection is age dependent. Epidemiol Infect. 1999;123:479-86.

12. Gambhir M, Blake IM, Basáñez M. Modelling trachoma : infection, transmission and control A report prepared for the International Trachoma Initiative.

13. Burton MJ, Hu VH, Massae P, Burr SE, Chevallier C, Afwamba IA, et al. What is causing active trachoma? The role of nonchlamydial bacterial pathogens in a low prevalence setting. Invest Ophthalmol Vis Sci. 2011;52:6012-7.

14. Burr SE, Hart JD, Edwards T, Baldeh I, Bojang E, Harding-Esch EM, et al. Association between ocular bacterial carriage and follicular trachoma following mass azithromycin distribution in The Gambia. PLoS Negl Trop Dis. 2013;7:e2347.

15. Dean D, Kandel RP, Adhikari HK, Hessel T. Multiple Chlamydiaceae species in trachoma: implications for disease pathogenesis and control. PLOS Med. 2008;5:e14.

16. Lietman T, Brooks D, Moncada J, Schachter J, Dawson C, Dean D. Chronic follicular conjunctivitis associated with Chlamydia psittaci or Chlamydia pneumoniae. Clin Infect Dis. 1998;26:1335-40.

17. Dean D, Rothschild J, Ruettger A, Kandel RP, Sachse K. Zoonotic Chlamydiaceae species associated with trachoma, Nepal. Emerg Infect Dis 2013:19:1948-55.

18. Harding-Esch EM, Sillah A, Edwards T, Burr SE, Hart JD, Joof H, et al. Mass treatment with azithromycin for trachoma: when is one round enough? Results from the PRET Trial in the Gambia. PLoS Negl Trop Dis. 2013;7:e2115.

19. Harding-Esch E, Jofre-Bonet M, Dhanjal JK, Burr S, Edwards T, Holland M, et al. Costs of testing for ocular Chlamydia trachomatis infection compared to mass drug administration for trachoma in the Gambia: application of results from the PRET study. PLoS Negl Trop Dis. 2015;9:e0003670.

20. Assaad FA, Maxwell-Lyons F. The use of catalytic models as tools for elucidating the clinical and epidemiological features of trachoma. Bull World Health Organ. 1966;34:341-55.

21. Duke-Elder W Textbook of Ophthalmology. Volume II: Clinical Methods of Examination, Congenital and Developmental Abnormalities, General Pathological and Therapeutic Considerations, Diseases of the Outer Eye. London, England: Henry Kimpton; 1937

22. Woodhouse DF. Some problems of trachoma diagnosis. Trans Ophthalmol Soc U K. 1973;93:635-40.

23. WHO simplified trachoma grading system. Community Eye Health 2004, 17:68.

24. Dawson CR, Jones BR. TM: Guide to Trachoma Control. Geneva: World Health Organization; 1981.

25. Last AR, Burr SE, Weiss HA, Harding-Esch EM, Cassama E, Nabicassa M, et al. Risk Factors for Active Trachoma and Ocular Chlamydia trachomatis Infection in Treatment-Naïve Trachoma-Hyperendemic Communities of the Bijagós Archipelago, Guinea Bissau. PLoS Negl Trop Dis. 2014;8, e2900.

26. Roberts CH, Last A, Molina-Gonzalez S, Cassama E, Butcher R, Nabicassa M, et al. Development and evaluation of a next-generation digital PCR diagnostic assay for ocular Chlamydia trachomatis infections. J Clin Microbiol. 2013;51:2195-203.

27. Last AR, Roberts CH, Cassama E, Nabicassa M, Molina-Gonzalez S, Burr SE, et al. Plasmid copy number and disease severity in naturally occurring ocular Chlamydia trachomatis infection. J Clin Microbiol. 2014;52:324-7.

\section{Submit your next manuscript to BioMed Central and we will help you at every step:}

- We accept pre-submission inquiries

- Our selector tool helps you to find the most relevant journal

- We provide round the clock customer support

- Convenient online submission

- Thorough peer review

- Inclusion in PubMed and all major indexing services

- Maximum visibility for your research

Submit your manuscript at www.biomedcentral.com/submit 